\title{
Modified Homotopy Perturbation Method for Solving Fractional Differential Equations
}

\author{
A. A. Hemeda \\ Department of Mathematics, Faculty of Science, Tanta University, Tanta 31527, Egypt \\ Correspondence should be addressed to A. A. Hemeda; aahemeda@yahoo.com
}

Received 29 June 2014; Accepted 22 October 2014; Published 26 November 2014

Academic Editor: Md Sazzad Hossien Chowdhury

Copyright ( 2014 A. A. Hemeda. This is an open access article distributed under the Creative Commons Attribution License, which permits unrestricted use, distribution, and reproduction in any medium, provided the original work is properly cited.

\begin{abstract}
The modified homotopy perturbation method is extended to derive the exact solutions for linear (nonlinear) ordinary (partial) differential equations of fractional order in fluid mechanics. The fractional derivatives are taken in the Caputo sense. This work will present a numerical comparison between the considered method and some other methods through solving various fractional differential equations in applied fields. The obtained results reveal that this method is very effective and simple, accelerates the rapid convergence of the series solution, and reduces the size of work to only one iteration.
\end{abstract}

\section{Introduction}

To find the explicit solutions of linear (nonlinear) differential equations, many powerful methods have been used. The homotopy perturbation method (HPM) [1] was first proposed by He in 1998. The application of the HPM [1-18] has appeared in a lot of researches, especially during recent years, which show that the method is a powerful technique for studying the numerical solutions.

Motivated by the above works [1-18], we would like to extend the application of the modified homotopy perturbation method (MHPM) to collection of linear (nonlinear) ordinary (partial) differential equations of fractional derivative in applied fields such as fractional wave equation, fractional Burgers' equation, fractional Klein-Gordon equation, and Bagley-Torvik equation and other equations. This modification demonstrates a rapid convergence of the series solution if it compared with standard HPM, variational iteration method (VIM), Adomian decomposition method (ADM), and new Jacobi operational matrix (JOM) [19-27] and, therefore, it has been shown to be computationally efficient in several examples in applied fields. In addition, the modified algorithm may give the exact solution for the fractional differential equations by using one iteration only. The obtained results suggest that this method introduces a powerful improvement for solving fractional differential equations.

\section{Basic Definitions}

Fractional calculus unifies and generalizes the notations of integer-order differentiation and $n$-fold integration [28, 29]. We give some basic definitions and properties of fractional calculus theory which will be used in this paper.

Definition 1 (see $[28,29]$ ). A real function $f(x), x>0$ is said to be in the space $C_{\mu}, \mu \in R$ if there exists a real number $p(>\mu)$, such that $f(x)=x^{p} f_{1}(x)$, where $f_{1}(x) \in C[0, \infty)$, and it is said to be in the space $C_{\mu}^{m}$ if and only if $f^{(m)} \in C_{m}$, $m \in N$.

The Riemann-Liouville fractional integral operator is defined as follows.

Definition 2 (see $[28,29])$. The Riemann-Liouville integral operator of order $\alpha \geq 0$ of a function $f \in C_{\mu}, \mu \geq-1$, is defined as

$$
\begin{aligned}
I^{\alpha} f(x) & =\frac{1}{\Gamma(\alpha)} \int_{0}^{x}(x-t)^{\alpha-1} f(t) d t, \quad \alpha>0, x>0, \\
I^{0} f(x) & =f(x) .
\end{aligned}
$$

In this paper only real and positive values of $\alpha$ will be considered. 
Properties of the operator $I^{\alpha}$ can be found in [29] and we mention only
(1) $I^{\alpha} I^{\beta} f(x)=I^{\alpha+\beta} f(x)$,
(2) $I^{\alpha} I^{\beta} f(x)=I^{\beta} I^{\alpha} f(x)$,
(3) $I^{\alpha} x^{\gamma}=\Gamma(\gamma+1) / \Gamma(\gamma+1+\alpha)$.

Definition 3 (see $[28,29]$ ). The fractional derivative of $f(x)$ in Caputo sense is defined as

$$
\begin{aligned}
D^{\alpha} f(x) & =I^{m-\alpha} D^{m} f(x) \\
& =\frac{1}{\Gamma(m-\alpha)} \int_{0}^{x}(x-t)^{m-\alpha-1} f^{(m)}(t) d t,
\end{aligned}
$$

for $m-1<\alpha \leq m, m \in N, x>0, f \in C_{-1}^{m}$.

Definition 4 (see $[28,29]$ ). For $m$ to be the smallest integer that exceeds $\alpha$, the Caputo time-fractional derivative of order $\alpha>0$ is defined as

$$
\begin{aligned}
& D_{t}^{\alpha} f(x, t) \\
& =\left\{\begin{array}{l}
\frac{1}{\Gamma(m-\alpha)} \int_{0}^{t}(t-\tau)^{m-\alpha-1} \frac{\partial^{m}}{\partial \tau^{m}} f(x, \tau) d \tau, \\
\quad m-1<\alpha \leq m, \\
\frac{\partial^{m}}{\partial t^{m}} f(x, t), \quad \alpha=m, \quad m \in N .
\end{array}\right.
\end{aligned}
$$

\section{Homotopy Perturbation Method}

In this section, we will present a review of the HPM and the MHPM.

The principles of the HPM and its applicability for various kinds of differential equations are given in many records [1$10,30,31]$. Consider the nonlinear differential equation:

$$
L(u)+N(u)=f(r), \quad r \in \Omega,
$$

with boundary conditions:

$$
B\left(u, \frac{\partial u}{\partial n}\right)=0, \quad r \in \Gamma .
$$

The He's homotopy perturbation technique defines the homotopy $v(r, p): \Omega \times[0,1] \rightarrow R$, which satisfies

$$
\begin{aligned}
H(v, p)= & (1-p)\left[L(v)-L\left(u_{0}\right)\right] \\
& +p[L(v)+N(v)-f(r)]=0
\end{aligned}
$$

or

$$
H(v, p)=L(v)-L\left(u_{0}\right)+p L\left(u_{0}\right)+p[N(v)-f(r)]=0,
$$

where $r \in \Omega$ and $p \in[0,1]$ is an impeding parameter, $u_{0}$ is an initial approximation which satisfies the boundary conditions, and $n$ is the unit outward normal of $\Omega$. Obviously, from (5) and (6), we have

$$
\begin{gathered}
H(v, 0)=L(v)-L\left(u_{0}\right)=0, \\
H(v, 1)=L(v)+N(v)-f(r)=0 .
\end{gathered}
$$

The changing process of $p$ from zero to unity is just that of $v(r, p)$ from $u_{0}$ to $u(r)$. In topology, this so-called deformation, $L(v)-L\left(u_{0}\right)$, and $L(v)+N(v)-f(r)$ are homotopic. The basic assumption is that the solution of (5) and (6) can be expressed as a power series in $p$ :

$$
v=v_{0}+p v_{1}+p^{2} v_{2}+\cdots \text {. }
$$

The approximate solution of (4a) and (4b), therefore, can be readily obtained:

$$
u=\lim _{p \rightarrow 1} v=v_{0}+v_{1}+v_{2}+\cdots
$$

The series (9) is convergent for most cases, and the rate of convergence depends on $A(u)=L(u)+N(u)$ [17].

3.1. Modified Homotopy Perturbation Method. The modified form of the HPM proposed by Odibat [11] can be established based on the assumption that the function $f(r)$ in (4a) can be divided into two parts, namely, $f_{0}$ and $f_{1}$, as

$$
f(r)=f_{0}(r)+f_{1}(r) .
$$

According to this assumption, $f(r)=f_{0}(r)+f_{1}(r)$, we can construct the homotopy $v(r, p): \Omega \times[0,1] \rightarrow R$, which satisfies

$$
\begin{aligned}
H(v, p)= & (1-p)\left[L(v)-L\left(u_{0}\right)\right] \\
& +p\left[L(v)+N(v)-f_{1}(r)\right]=f_{0}(r),
\end{aligned}
$$

or

$$
\begin{aligned}
H(v, p)= & L(v)-L\left(u_{0}\right)+p L\left(u_{0}\right) \\
& +p\left[N(v)-f_{1}(r)\right]=f_{0}(r) .
\end{aligned}
$$

Here, a slight variation was proposed only on the components $u_{0}$ and $u_{1}$. The suggestion was that only the part $f_{0}$ be assigned to zeroth component $u_{0}$, whereas the remaining part $f_{1}$ be combined with the component $u_{1}$. If we set $f_{1}(r)=f(r)$ and $f_{0}(r)=0$, then the homotopy (11) or (12) reduces to the homotopy (5) or (6), respectively. However the success of the method depends on the proper selection of the functions $f_{0}$ and $f_{1}$.

3.2. Reliable Algorithm. We introduce a reliable algorithm to handle in a realistic and efficient way the linear (nonlinear) partial differential equations of fractional order. Consider the general fractional differential equation:

$$
\begin{array}{r}
D_{t}^{\alpha} u(x, t)=L\left(u, u_{x}, u_{x x}\right)+N\left(u, u_{x}, u_{x x}\right)+f(x, t), \\
t>0,
\end{array}
$$

where $L$ is a linear operator, $N$ is a nonlinear operator, $f$ is a known analytic function, and $D^{\alpha}, m-1<\alpha<m$, is the Caputo fractional derivative of order $\alpha$, subject to the initial conditions:

$$
u^{(k)}(x, 0)=h_{k}(x), \quad k=0,1,2, \ldots, m-1 .
$$


In view of the homotopy technique, we can construct the following homotopy:

$$
D_{t}^{\alpha} u-L\left(u, u_{x}, u_{x x}\right)-f(x, t)=p\left[N\left(u, u_{x}, u_{x x}\right)\right],
$$

or

$$
D_{t}^{\alpha} u-f(x, t)=p\left[L\left(u, u_{x}, u_{x x}\right)+N\left(u, u_{x}, u_{x x}\right)\right] .
$$

In view of the modified homotopy technique, if we write $f(x, t)=f_{0}(x, t)+f_{1}(x, t)$, we can construct the following homotopy:

$$
\begin{aligned}
D_{t}^{\alpha} u & -L\left(u, u_{x}, u_{x x}\right)-f_{0}(x, t) \\
& =p\left[N\left(u, u_{x}, u_{x x}\right)+f_{1}(x, t)\right],
\end{aligned}
$$

or

$$
\begin{aligned}
& D_{t}^{\alpha} u-f_{0}(x, t) \\
& \quad=p\left[L\left(u, u_{x}, u_{x x}\right)+N\left(u, u_{x}, u_{x x}\right)+f_{1}(x, t)\right],
\end{aligned}
$$

where $p \in[0,1]$. The homotopy parameter $p$ always changes from zero to unity. The basic assumption is that the solution of (14) or (15) and (16) or (17) can be written as a power series in $p$ :

$$
u=u_{0}+p u_{1}+p u_{2}+\cdots .
$$

Finally, we approximate the solution $u(x, t)=$ $\sum_{n=0}^{\infty} u_{n}(x, t)$ by the $n$-term truncated series:

$$
u_{N}(x, t)=\sum_{n=0}^{N-1} u_{n}(x, t) .
$$

Also, for solving fractional physical differential equations, you can see [32].

\section{Applications}

4.1. Linear Problems. To incorporate the above discussion, four linear examples will be studied. The MHPM is used to obtain the exact solutions of the problems.

Example 5. Consider the following one-dimensional linear inhomogeneous fractional wave equation:

$$
\begin{array}{r}
D_{t}^{\alpha} u+u_{x}=\frac{t^{1-\alpha}}{\Gamma(2-\alpha)} \sin x+t \cos x, \\
t>0, \quad x \in R, \quad 0<\alpha \leq 1,
\end{array}
$$

subject to the initial condition:

$$
u(x, 0)=0 .
$$

According to the ADM [22], the 3-term solution in series form is given by

$$
\begin{aligned}
u(x, t)= & t \sin x+\frac{t^{1+\alpha}}{\Gamma(2+\alpha)} \cos x \\
& -\frac{t^{1+\alpha}}{\Gamma(2+\alpha)} \cos x+\frac{t^{1+2 \alpha}}{\Gamma(2+2 \alpha)} \sin x \\
& -\frac{t^{1+2 \alpha}}{\Gamma(2+2 \alpha)} \sin x-\frac{t^{1+3 \alpha}}{\Gamma(2+3 \alpha)} \cos x+\cdots
\end{aligned}
$$

According to the VIM [22], if we begin with $u_{0}=0$, the $u_{3}$ solution in series form is given by

$$
\begin{aligned}
u(x, t)= & t \sin x+\frac{t^{1+\alpha}}{\Gamma(2+\alpha)} \cos x-\frac{t^{1+\alpha}}{\Gamma(2+\alpha)} \cos x \\
& +\frac{t^{1+2 \alpha}}{\Gamma(2+2 \alpha)} \sin x-\frac{t^{1+2 \alpha}}{\Gamma(2+2 \alpha)} \sin x \\
& -\frac{t^{1+3 \alpha}}{\Gamma(2+3 \alpha)} \cos x+\cdots .
\end{aligned}
$$

In both series, it is easily observed that the self-canceling "noise" terms appear between various components. Canceling the noise terms and keeping the nonnoise terms in both series yields the exact solution of (20a) and (20b) given by

$$
u(x, t)=t \sin x .
$$

In the VIM, if we begin with $u_{0}=t \sin x$, then the exact solution follows immediately by using two iterations only.

According to the MHPM, in view of (17), the homotopy for (20a) and (20b) can be constructed as

$$
D_{t}^{\alpha} u-\frac{t^{1-\alpha}}{\Gamma(2-\alpha)} \sin x=p\left[t \cos x-u_{x}\right],
$$

where $f_{0}=t^{1-\alpha} / \Gamma(2-\alpha) \sin x, f_{1}=t \cos x$. Substituting (18) and the initial condition (20b) into (24) and equating the terms with identical powers of $p$, we obtain the following set of equations:

$$
\begin{aligned}
& p^{0}: D_{t}^{\alpha} u_{0}=\frac{t^{1-\alpha}}{\Gamma(2-\alpha)} \sin x, \quad u_{0}(x, 0)=0, \\
& p^{1}: D_{t}^{\alpha} u_{1}=t \cos x-\left(u_{0}\right)_{x}, \quad u_{1}(x, 0)=0, \\
& p^{2}: D_{t}^{\alpha} u_{2}=-\left(u_{1}\right)_{x}, \quad u_{2}(x, 0)=0,
\end{aligned}
$$

Consequently, the first few components of the modified homotopy perturbation solution for (20a) and (20b) are derived as follows:

$$
u_{0}(x, t)=t \sin x, \quad u_{j}(x, t)=0, \quad j \geq 1 .
$$

The exact solution $u(x, t)=t \sin x$ follows immediately. The success of obtaining the exact solution by one iteration only is a result of the proper selection of $f_{0}(r)$ and $f_{1}(r)$, which means that the MHPM is a powerful method compared with the other two methods.

Example 6. In this example we consider the one-dimensional linear inhomogeneous fractional Burgers equation given by

$$
\begin{array}{r}
D_{t}^{\alpha} u+u_{x}-u_{x x}=\frac{2 t^{2-\alpha}}{\Gamma(3-\alpha)}+2(x-1), \\
t>0, \quad x \in R, \quad 0<\alpha \leq 1,
\end{array}
$$


subject to the initial condition:

$$
u(x, 0)=x^{2} .
$$

By ADM [22], the first few components of solution are

$$
\begin{aligned}
& u_{0}(x, t)=x^{2}+t^{2}+\frac{2 t^{\alpha}}{\Gamma(1+\alpha)}(x-1), \\
& u_{1}(x, t)=-\frac{2 t^{\alpha}}{\Gamma(1+\alpha)}(x-1)-\frac{2 t^{\alpha}}{\Gamma(1+2 \alpha)}, \\
& u_{2}(x, t)=-\frac{2 t^{\alpha}}{\Gamma(1+2 \alpha)}, \\
& u_{3}(x, t)=0
\end{aligned}
$$

also as a result $u_{j}(x, t)=0, j \geq 3$. The exact solution is therefore given by

$$
u(x, t)=x^{2}+t^{2} .
$$

By VIM [22], if we begin with $u_{0}=x^{2}$, we can obtain

$$
u_{1}(x, t)=x^{2}+t^{2}, \ldots, \quad u_{n}(x, t)=x^{2}+t^{2} .
$$

The exact solution $u(x, t)=x^{2}+t^{2}$ follows immediately. The success of obtaining the exact solution by using two iterations is a result of the proper selection of initial guess $u_{0}$.

By MHPM, in view of (17), the homotopy for (27a) and (27b) can be constructed as

$$
D_{t}^{\alpha} u-\frac{2 t^{2-\alpha}}{\Gamma(3-\alpha)}=p\left[2(x-1)-u_{x}+u_{x x}\right],
$$

where $f_{0}=2 t^{2-\alpha} / \Gamma(3-\alpha), f_{1}=2(x-1)$. Substituting (18) and the initial condition (27b) into (31) and equating the terms with equal powers of $p$, we obtain the following set of equations:

$$
\begin{aligned}
& p^{0}: D_{t}^{\alpha} u_{0}=\frac{2 t^{2-\alpha}}{\Gamma(3-\alpha)}, \quad u_{0}(x, 0)=x^{2}, \\
& p^{1}: D_{t}^{\alpha} u_{1}=2(x-1)-\left(u_{0}\right)_{x}+\left(u_{0}\right)_{x x}, \quad u_{1}(x, 0)=0, \\
& p^{2}: D_{t}^{\alpha} u_{2}=-\left(u_{1}\right)_{x}+\left(u_{1}\right)_{x x}, \quad u_{2}(x, 0)=0,
\end{aligned}
$$

Consequently, the first few components of the modified homotopy perturbation solution for (27a) and (27b) are derived as follows:

$$
u_{0}(x, t)=x^{2}+t^{2}, \quad u_{j}(x, t)=0, \quad j \geq 1 .
$$

The exact solution $u(x, t)=x^{2}+t^{2}$ follows immediately. The success of obtaining the exact solution by using one iteration only is a result of the proper selection of $f_{0}(r)$ and $f_{1}(r)$.
Example 7. In this example, we consider the one-dimensional linear inhomogeneous fractional Klein-Gordon equation given by

$$
\begin{array}{r}
D_{t}^{\alpha} u-u_{x x}+u=6 x^{3} \frac{t^{3-\alpha}}{\Gamma(4-\alpha)}+\left(x^{3}-6 x\right) t^{3}, \\
t>0, \quad x \in R, \quad 1<\alpha \leq 2,
\end{array}
$$

subject to the initial conditions:

$$
u(x, 0)=0, \quad u_{t}(x, 0)=0 .
$$

The 2-term solution, according to ADM [22], in series form is given by

$$
\begin{aligned}
u(x, t)= & 6 x^{3} \frac{t^{1+\alpha}}{\Gamma(2+\alpha)}+6\left(x^{3}-6 x\right) \frac{t^{3+\alpha}}{\Gamma(4+\alpha)} \\
& +36 x \frac{t^{1+2 \alpha}}{\Gamma(2+2 \alpha)}+36 x \frac{t^{3+2 \alpha}}{\Gamma(4+2 \alpha)} \\
& -6 x^{3} \frac{t^{1+2 \alpha}}{\Gamma(2+2 \alpha)}-6\left(x^{3}-6 x\right) \frac{t^{3+2 \alpha}}{\Gamma(4+\alpha)} \\
& +\cdots,
\end{aligned}
$$

and the $u_{2}$ solution according to VIM [22] in series form is given by

$$
\begin{aligned}
u_{2}(x, t)=6 x^{3} & \frac{t^{1+\alpha}}{\Gamma(2+\alpha)}+6\left(x^{3}-6 x\right) \frac{t^{3+\alpha}}{\Gamma(4+\alpha)}-(\alpha-1)^{2} \\
\cdot & {\left[6\left(x^{3}-6 x\right) \frac{t^{1+2 \alpha}}{\Gamma(2+2 \alpha)}\right.} \\
+ & \left.6\left(x^{3}-12 x\right) \frac{t^{3+2 \alpha}}{\Gamma(4+2 \alpha)}\right]+\cdots .
\end{aligned}
$$

From (35) and (36), the ADM and the VIM give the same solution for the classical Klein-Gordon equation (34a) and (34b) in case $\alpha=2$ which is given by

$$
\begin{aligned}
u(x, t)= & x^{3} t^{3}+6\left(x^{3}-6 x\right) \frac{t^{5}}{\Gamma(6)} \\
& +36 x \frac{t^{5}}{\Gamma(6)}-36 x \frac{6 t^{7}}{\Gamma(8)}-6 x^{3} \frac{t^{5}}{\Gamma(6)} \\
& -6\left(x^{3}-6 x\right) \frac{t^{7}}{\Gamma(8)}+\cdots
\end{aligned}
$$

Canceling the noise terms and keeping the nonnoise terms in (37) yields the exact solution of (34a) and (34b), for the special case $\alpha=2$ :

$$
u(x, t)=x^{3} t^{3},
$$

which is easily verified. 
By the MHPM, in view of (17), the homotopy for (34a) and (34b) becomes

$$
D_{t}^{\alpha} u-6 x^{3} \frac{t^{3-\alpha}}{\Gamma(4-\alpha)}=p\left[\left(x^{3}-6 x\right) t^{3}+u_{x x}-u\right]
$$

where $f_{0}=6 x^{3}\left(t^{3-\alpha} / \Gamma(4-\alpha)\right), f_{1}=\left(x^{3}-6 x\right) t^{3}$. Substituting (18) and the initial conditions (34b) into (39) and equating the terms with equal powers of $p$, we obtain the following set of equations:

$$
\begin{gathered}
p^{0}: D_{t}^{\alpha} u_{0}=6 x^{3} \frac{t^{3-\alpha}}{\Gamma(4-\alpha)}, \quad u_{0}(x, 0)=0,\left(u_{0}\right)_{t}(x, 0)=0, \\
p^{1}: D_{t}^{\alpha} u_{1}=\left(x^{3}-6 x\right) t^{3}+\left(u_{0}\right)_{x x}-u_{0}, \\
u_{1}(x, 0)=0, \quad\left(u_{1}\right)_{t}(x, 0)=0, \\
p^{2}: D_{t}^{\alpha} u_{2}=\left(u_{1}\right)_{x x}-u_{1}, \quad u_{2}(x, 0)=0,\left(u_{2}\right)_{t}(x, 0)=0,
\end{gathered}
$$

By solving the above set of equations, the first few components of the modified homotopy perturbation solution for (34a) and (34b) are

$$
u_{0}(x, t)=x^{3} t^{3}, \quad u_{j}(x, t)=0, \quad j \geq 1
$$

The exact solution $u(x, t)=x^{3} t^{3}$ follows immediately.

Example 8. In this example, we consider the initial value problem in the case of the inhomogeneous Bagley-Torvik equation:

$$
D^{2} u(x)+D^{1.5} u+u=g(x), \quad x \in[0, L],
$$

subject to the initial conditions:

$$
u(0)=1, \quad u_{x}(0)=1
$$

where $g(x)=1+x$.

To solve (42a) and (42b) by applying the shifted Jacobi polynomials, with $N=2$, we may write the approximate solution $u(x)$ and the right hand side $g(x)$ in the forms [21]

$$
\begin{aligned}
& u(x)=\sum_{i=0}^{2} c_{i} P_{L, i}^{(\alpha, \beta)}(x)=C^{T} \phi(x), \\
& g(x)=\sum_{i=0}^{2} g_{i} P_{L, i}^{(\alpha, \beta)}(x)=G^{T} \phi(x) .
\end{aligned}
$$

Here, the operational matrices corresponding to (42a) and (42b) can be written as follows:

$$
\begin{gathered}
D^{2}=\left(\begin{array}{ccc}
0 & 0 & 0 \\
0 & 0 & 0 \\
\Delta_{2}(2,0) & \Delta_{2}(2,1) & \Delta_{2}(2,2)
\end{array}\right), \\
D^{1.5}=\left(\begin{array}{ccc}
0 & 0 & 0 \\
0 & 0 & 0 \\
\Delta_{1.5}(2,0) & \Delta_{1.5}(2,1) & \Delta_{1.5}(2,2)
\end{array}\right), \\
G=\left(\begin{array}{l}
g_{0} \\
g_{1} \\
g_{2}
\end{array}\right) .
\end{gathered}
$$

Application of the tan method for (42a) and (42b) gives

$$
\begin{gathered}
c_{0}+\left(\Delta_{2}(2,0)+\Delta_{1.5}(2,0)\right) c_{2}-g_{0}=0, \\
c_{0}-(\beta+1) c_{1}+\frac{(\beta+1)(\beta+2)}{2} c_{2}-1=0, \\
(\alpha+\beta+2) c_{1}-(\beta+2)(\alpha+\beta+3) c_{2}-L=0 .
\end{gathered}
$$

By solving the above linear algebraic equations, the approximate solution for (42a) and (42b) can be written as

$$
u(x)=\left(\begin{array}{lll}
c_{0} & c_{1} & c_{2}
\end{array}\right)\left(\begin{array}{c}
P_{L, 0}^{(\alpha, \beta)}(x) \\
P_{L, 1}^{(\alpha, \beta)}(x) \\
P_{L, 2}^{(\alpha, \beta)}(x)
\end{array}\right)=1+x
$$

which is the exact solution of (42a) and (42b).

By the MHPM, the homotopy for (42a) and (42b) can be written as

$$
D^{2} u(x)=p\left[1+x-D^{1.5} u-u\right] .
$$

Substituting (18) and the initial conditions (42b) into (47), as the above examples, we can obtain the following set of equations:

$$
\begin{aligned}
& p^{0}: D^{2} u_{0}=0, \quad u_{0}(0)=1, u_{0}^{\prime}=1, \\
& p^{1}: D^{2} u_{1}=1+x-D^{1.5} u_{0}-u_{0}, \quad u_{1}(0)=0, u_{1}^{\prime}=0, \\
& p^{2}: D^{2} u_{2}=-D^{1.5} u_{1}-u_{1}, \quad u_{2}(0)=0, u_{2}^{\prime}=0,
\end{aligned}
$$

Solving the above set of equations, the first few components of the modified homotopy perturbation solution for (42a) and (42b) are

$$
u_{0}(x)=1+x, \quad u_{j}(x)=0, \quad j \geq 1 .
$$

The exact solution $u(x)=1+x$ follows immediately. In this example, it is clear that the computational procedures of the present method are simple, easy and short than the other method. 
4.2. Nonlinear Problems. For nonlinear problems, there exists no method that yields exact solutions and therefore only approximate solutions can be derived. In this subsection the MHPM is used to obtain the exact solution for four nonlinear problems.

Example 9. In this example, we consider the nonlinear timefractional advection partial differential equation:

$$
D_{t}^{\alpha} u+u u_{x}=x+x t^{2}, \quad t>0, x \in R, 0<\alpha \leq 1,
$$

subject to the initial condition:

$$
u(x, 0)=0 .
$$
is [19]

In view of HPM, (15), the homotopy for (50a) and (50b)

$$
\frac{\partial u}{\partial t}-x-x t^{2}=p\left[\frac{\partial u}{\partial t}-u u_{x}-D_{t}^{\alpha} u\right] .
$$

Substituting (18) and the initial condition (50b) into (51) and equating the terms with equal powers of $p$, we obtain the set of equations

$$
\begin{gathered}
p^{0}: \frac{\partial u_{0}}{\partial t}=x+x t^{2}, \quad u_{0}(x, 0)=0, \\
p^{1}: \frac{\partial u_{1}}{\partial t}=\frac{\partial u_{0}}{\partial t}-u_{0}\left(u_{0}\right)_{x}-D_{t}^{\alpha} u_{0}, \quad u_{1}(x, 0)=0, \\
p^{2}: \frac{\partial u_{2}}{\partial t}=\frac{\partial u_{1}}{\partial t}-u_{0}\left(u_{1}\right)_{x}-u_{1}\left(u_{0}\right)_{x}-D_{t}^{\alpha} u_{1}, \\
u_{2}(x, 0)=0,
\end{gathered}
$$

Solving the above set of equations, the first few components of the homotopy perturbation solution for (50a) and (50b) are

$$
\begin{aligned}
& u_{0}(x, t)=x\left(t+\frac{t^{3}}{3}\right) \\
& u_{1}(x, t)=x\left(t-\frac{2 t^{5}}{15}-\frac{t^{7}}{63}-\frac{t^{2-\alpha}}{\Gamma(3-\alpha)}-\frac{2 t^{4-\alpha}}{\Gamma(5-\alpha)}\right),
\end{aligned}
$$

The 3-term approximate solution for the homotopy perturbation solution for (50a) and (50b) in series form is

$$
\begin{aligned}
u(x, t)=x[ & 3 t-\frac{4 t^{5}}{15}+\frac{2 t^{7}}{315}+\frac{2 t^{9}}{567}-\frac{4 t^{11}}{2475} \\
& -\frac{4 t^{13}}{12285}-\frac{t^{15}}{59535}-\frac{3 t^{2-\alpha}}{\Gamma(3-\alpha)}+\frac{t^{3-2 \alpha}}{\Gamma(4-2 \alpha)} \\
& +\left(\frac{2}{\Gamma(3-\alpha)}-\frac{4}{\Gamma(4-\alpha)}\right) \frac{t^{4-\alpha}}{(4-\alpha)} \\
& \left.+\left(\frac{4}{\Gamma(5-\alpha)}+\frac{16}{\Gamma(6-\alpha)}\right) \frac{t^{6-\alpha}}{(6-\alpha)}+\cdots\right]
\end{aligned}
$$

In view of MHPM, (17), the homotopy for (50a) and (50b) is

$$
D_{t}^{\alpha} u-x=p\left[x t^{2}-u u_{x}\right]
$$

Substituting (18) and the initial condition of (50b) into (55), as above, we have

$$
\begin{aligned}
& p^{0}: D_{t}^{\alpha} u_{0}=x, \quad u_{0}(x, 0)=0, \\
& p^{1}: D_{t}^{\alpha} u_{1}=x t^{2}-u_{0}\left(u_{0}\right)_{x}, \quad u_{1}(x, 0)=0, \\
& p^{2}: D_{t}^{\alpha} u_{2}=-u_{1}\left(u_{0}\right)_{x}-u_{0}\left(u_{1}\right)_{x}, \quad u_{2}(x, 0)=0,
\end{aligned}
$$

Solving the above set of equations, we have the following first few components of the modified homotopy perturbation solution for (50a) and (50b):

$$
u_{0}(x, t)=x t, \quad u_{j}(x, t)=0, \quad j \geq 1 \text {. }
$$

The exact solution $u(x, t)=x t$ follows immediately.

Example 10. Consider the nonlinear initial value problem with fractional order:

$$
D^{3} u(x)+D^{2.5} u(x)+u^{2}(x)=x^{4}
$$

subject to the initial conditions:

$$
u(0)=0, \quad u^{\prime}(0)=0, \quad u^{\prime \prime}(0)=2 .
$$

Applying the shifted Jacobi collocation method with the shifted Jacobi operational matrix technique [21] with $N=3$, the approximate solution $u(x)$ can be written in the form

$$
u(x)=\sum_{i=0}^{3} c_{i} P_{L, i}^{(\alpha, \beta)}(x)=C^{T} \phi(x) .
$$

Regarding problem (58a) and (58b), the corresponding Jacobi operational matrices can be written as

$$
\begin{gathered}
D^{3}=\left(\begin{array}{cccc}
0 & 0 & 0 & 0 \\
0 & 0 & 0 & 0 \\
0 & 0 & 0 & 0 \\
\Delta_{3}(3,0) & \Delta_{3}(3,1) & \Delta_{3}(3,2) & \Delta_{3}(3,3)
\end{array}\right), \\
D^{2.5}=\left(\begin{array}{cccc}
0 & 0 & 0 & 0 \\
0 & 0 & 0 & 0 \\
0 & 0 & 0 & 0 \\
\Delta_{2.5}(3,0) & \Delta_{2.5}(3,1) & \Delta_{2.5}(3,2) & \Delta_{2.5}(3,3)
\end{array}\right), \\
C=\left(\begin{array}{c}
c_{0} \\
c_{1} \\
c_{2} \\
c_{3}
\end{array}\right) .
\end{gathered}
$$


As in Example 8, the application of the tan method for (58a) and (58b) gives

$$
\begin{aligned}
C^{T} \phi(0)= & c_{0}-(\beta+1) c_{1}+\frac{(\beta+1)(\beta+2)}{2} c_{2} \\
& -\frac{(\beta+1)(\beta+2)(\beta+3)}{6} c_{3}=0, \\
C^{T} D^{(1)} \phi(0)= & (\alpha+\beta+2) c_{1}-(\beta+2)(\alpha+\beta+3) c_{2} \\
& +\frac{(\beta+2)(\beta+3)(\alpha+\beta+4)}{2} c_{3}=0, \\
C^{T} D^{(2)} \phi(0)= & (\alpha+\beta+3)(\alpha+\beta+4) c_{2} \\
& -(\beta+3)(\alpha+\beta+4)(\alpha+\beta+5) c_{3}=2 L^{2} .
\end{aligned}
$$

By solving the above equations, the approximate solution for (58a) and (58b) can be written as

$$
u(x)=\left(\begin{array}{llll}
c_{0} & c_{1} & c_{2} & c_{3}
\end{array}\right)\left(\begin{array}{c}
P_{L, 0}^{(\alpha, \beta)}(x) \\
P_{L, 1}^{(\alpha, \beta)}(x) \\
P_{L, 2}^{(\alpha, \beta)}(x) \\
P_{L, 3}^{(\alpha, \beta)}(x)
\end{array}\right)=x^{2}
$$

which is the exact solution.

According to the MHPM, in view of (17), the homotopy for $(58 \mathrm{a})$ and $(58 \mathrm{~b})$ is

$$
D^{3} u=p\left[x^{4}-D^{2.5} u-u^{2}\right]
$$

As the above examples, we can obtain the following set of equations:

$$
\begin{gathered}
p^{0}: D^{3} u_{0}=0, \quad u_{0}(0)=0, \quad u_{0}^{\prime}(0)=0, u_{0}^{\prime \prime}(0)=2, \\
p^{1}: D^{3} u_{1}=x^{4}-D^{2.5} u_{0}-u_{0}^{2}, \\
u_{1}(0)=0, \quad u_{1}^{\prime}(0)=0, \quad u_{1}^{\prime \prime}(0)=0, \\
p^{2}: D^{3} u_{2}=-D^{2.5} u_{1}-2 u_{0} u_{1}, \\
u_{2}(0)=0, \quad u_{2}^{\prime}(0)=0, \quad u_{2}^{\prime \prime}(0)=0
\end{gathered}
$$

Solving the above equations, we have the following first few components of solution for (58a) and (58b):

$$
u_{0}(x)=x^{2}, \quad u_{j}(x)=0, \quad j \geq 1
$$

The exact solution $u(x)=x^{2}$ follows immediately.
TABLE 1: Maximum absolute error for $\xi=2.5, \eta=1.5$, and $\theta=0.9$ and different choices of $\alpha, \beta$, and $N$.

\begin{tabular}{lcccccc}
\hline$N$ & $\alpha$ & $\beta$ & Error & $\alpha$ & $\beta$ & Error \\
\hline 4 & & & $3.91 \times 10^{-3}$ & & & $1.84 \times 10^{-3}$ \\
8 & \multirow{2}{*}{0} & 0 & $1.42 \times 10^{-3}$ & & & $5.40 \times 10^{-4}$ \\
16 & & & $1.24 \times 10^{-4}$ & 1 & 1 & $1.03 \times 10^{-4}$ \\
24 & & & $3.37 \times 10^{-5}$ & & & $3.39 \times 10^{-5}$ \\
\hline 4 & & & $2.73 \times 10^{-3}$ & & & $1.27 \times 10^{-3}$ \\
8 & \multirow{3}{*}{0.5} & 0.5 & $8.66 \times 10^{-4}$ & & & $3.47 \times 10^{-4}$ \\
16 & & & $1.17 \times 10^{-4}$ & 1.5 & 1.5 & $8.98 \times 10^{-5}$ \\
24 & & & $3.50 \times 10^{-5}$ & & & $3.15 \times 10^{-5}$ \\
\hline
\end{tabular}

Example 11. Consider the initial value problem of multiterm nonlinear fractional differential equation:

$$
\begin{aligned}
& D^{\xi} u(x)+D^{\eta} u(x) \cdot D^{\theta} u(x)+u^{2}(x) \\
& =x^{6}+\frac{6 x^{3-\xi}}{\Gamma(4-\xi)}+\frac{36 x^{6-\eta-\theta}}{\Gamma(4-\eta) \Gamma(4-\theta)},
\end{aligned}
$$

where $\xi \in(2,3), \eta \in(1,2)$, and $\theta \in(0,1)$, subject to the initial conditions:

$$
u(0)=0, \quad u^{\prime}(0)=0, \quad u^{\prime \prime}(0)=0 .
$$

The exact solution of this problem is $u(x)=x^{3}$.

In Table 1, Doha et al. in [21] introduce the maximum absolute error, using the shifted Jacobi collocation method based on the shifted Jacobi operational matrix, at $\xi=2.5$, $\eta=1.5$, and $\theta=0.9$ with various choices of $\alpha, \beta$, and $N$. Also, the maximum absolute error for four different cases of $N, \xi$, $\eta$, and $\theta$ and $\alpha=\beta=1.5$ are shown in Table 2 . From Table 2 , we see that as $\xi, \eta$, and $\theta$ approach their integer values, the solution of the fractional differential equation approaches that of the integer-order differential equations and accordingly the approximate solutions will be more accurate.

According to the MHPM, in view of (17), the homotopy for $(66)$ is

$$
\begin{aligned}
& D^{\xi} u-\frac{6 x^{3-\xi}}{\Gamma(4-\xi)} \\
& \quad=p\left[x^{6}+\frac{36 x^{6-\eta-\theta}}{\Gamma(4-\eta) \Gamma(4-\theta)}-D^{\eta} u \cdot D^{\theta} u-u^{2}\right]
\end{aligned}
$$


TABLE 2: Maximum absolute error for $\alpha=\beta=1.5$ and different choices of $\xi, \eta, \theta$, and $N$.

\begin{tabular}{|c|c|c|c|c|c|c|c|c|}
\hline$N$ & $\xi$ & $\eta$ & $\theta$ & Error & $\xi$ & $\eta$ & $\theta$ & Error \\
\hline 4 & \multirow{4}{*}{2.000001} & \multirow{4}{*}{1.000001} & \multirow{4}{*}{0.000001} & $1.47 \times 10^{-9}$ & \multirow{4}{*}{2.75} & \multirow{4}{*}{1.75} & \multirow{4}{*}{0.75} & $2.02 \times 10^{-3}$ \\
\hline 8 & & & & $2.43 \times 10^{-10}$ & & & & $5.93 \times 10^{-4}$ \\
\hline 16 & & & & $2.62 \times 10^{-11}$ & & & & $2.40 \times 10^{-4}$ \\
\hline 24 & & & & $6.29 \times 10^{-12}$ & & & & $1.06 \times 10^{-4}$ \\
\hline 4 & \multirow{4}{*}{2.99} & \multirow{4}{*}{1.99} & \multirow{4}{*}{0.99} & $1.85 \times 10^{-4}$ & \multirow{4}{*}{2.9999} & \multirow{4}{*}{1.9999} & \multirow{4}{*}{0.9999} & $1.91 \times 10^{-6}$ \\
\hline 8 & & & & $5.32 \times 10^{-5}$ & & & & $5.46 \times 10^{-7}$ \\
\hline 16 & & & & $3.50 \times 10^{-5}$ & & & & $3.67 \times 10^{-7}$ \\
\hline 24 & & & & $1.95 \times 10^{-5}$ & & & & $2.06 \times 10^{-7}$ \\
\hline
\end{tabular}

As above, we have the set of equations

$$
\begin{gathered}
p^{0}: D^{\xi} u_{0}=\frac{6 x^{3-\xi}}{\Gamma(4-\xi)}, \quad u_{0}(0)=0, \quad u_{0}^{\prime}(0)=0, \quad u_{0}^{\prime \prime}(0)=0 \\
p^{1}: D^{\xi} u_{1}=x^{6}+\frac{36 x^{6-\eta-\theta}}{\Gamma(4-\eta) \Gamma(4-\theta)}-D^{\eta} u_{0} \cdot D^{\theta} u_{0}-u_{0}^{2} \\
u_{1}(0)=0, \quad u_{1}^{\prime}(0)=0, \quad u_{1}^{\prime \prime}(0)=0 \\
p^{2}: D^{\xi} u_{2}=-D^{\eta} u_{0} \cdot D^{\theta} u_{1}-D^{\eta} u_{1} \cdot D^{\theta} u_{0}-2 u_{0} u_{1} \\
u_{2}(0)=0, \quad u_{2}^{\prime}(0)=0, \quad u_{2}^{\prime \prime}(0)=0
\end{gathered}
$$

Solving the above set of equations, we have the following first few components of solution for (66):

$$
u_{0}(x)=x^{3}, \quad u_{j}(x)=0, \quad j \geq 1
$$

The exact solution $u(x)=x^{3}$ follows immediately.

Example 12. Consider the nonlinear time-fractional partial differential equation:

$$
\begin{array}{r}
D_{t}^{\alpha} u+u\left(u_{x}-1\right)=\frac{2 t^{2-\alpha}}{\Gamma(3-\alpha)} e^{x}+t^{4} e^{2 x}-t^{2} e^{x} \\
t>0, \quad x \in R, \quad 1<\alpha \leq 2,
\end{array}
$$

subject to the initial conditions:

$$
u(x, 0)=0, \quad u_{t}(x, 0)=0 .
$$

According to the MHPM, in view of (17), the homotopy for (71a) and (71b) is

$$
D_{t}^{\alpha} u-\frac{2 t^{2-\alpha}}{\Gamma(3-\alpha)} e^{x}=p\left[t^{4} e^{2 x}-t^{2} e^{x}-u\left(u_{x}-1\right)\right]
$$

As above, we have the following set of equations:

$$
\begin{gathered}
p^{0}: D_{t}^{\alpha} u_{0}=\frac{2 t^{2-\alpha}}{\Gamma(3-\alpha)} e^{x}, \quad u_{0}(x, 0)=0,\left(u_{0}\right)_{t}(x, 0)=0 \\
p^{1}: D_{t}^{\alpha} u_{1}=t^{4} e^{2 x}-t^{2} e^{x}-u_{0}\left(\left(u_{0}\right)_{x}-1\right) \\
u_{1}(x, 0)=0, \quad\left(u_{1}\right)_{t}(x, 0)=0 \\
p^{2}: D_{t}^{\alpha} u_{2}=-u_{0}\left(u_{1}\right)_{x}-u_{1}\left(u_{0}\right)_{x}-u_{1} \\
u_{2}(x, 0)=0, \quad\left(u_{2}\right)_{t}(x, 0)=0 \\
\vdots
\end{gathered}
$$

Solving the above set of equations, we have the following first few components of the modified homotopy perturbation solution for (71a) and (71b):

$$
u_{0}(x, t)=t^{2} e^{x}, \quad u_{j}(x, t)=0, \quad j \geq 1 .
$$

The exact solution $u(x, t)=t^{2} e^{x}$ follows immediately.

\section{Conclusion}

In this paper, the MHPM is applied to approximate solutions for some of linear (nonlinear) ordinary (partial) differential equations of fractional order in applied fields. The obtained results, which compared with those obtained by some other methods, show us that this method can obtain the exact solution by only one iteration. It may be concluded that the modified method is remarkably very effective, very simple, and very fast in convergence compared with the other methods for handling these kinds of problems.

\section{Conflict of Interests}

The author declares that there is no conflict of interests regarding the publication of this paper.

\section{References}

[1] J.-H. He, "An approximate solution technique depending on an artificial parameter: a special example," Communications in Nonlinear Science and Numerical Simulation, vol. 3, no. 2, pp. 92-97, 1998. 
[2] S. Abbasbandy, "Iterated He's homotopy perturbation method for quadratic Riccati differential equation," Applied Mathematics and Computation, vol. 175, no. 1, pp. 581-589, 2006.

[3] S. Abbasbandy, "Application of He's homotopy perturbation method to functional integral equations," Chaos, Solitons \& Fractals, vol. 31, no. 5, pp. 1243-1247, 2007.

[4] J.-H. He, "Application of homotopy perturbation method to nonlinear wave equations," Chaos, Solitons \& Fractals, vol. 26, pp. 695-700, 2005.

[5] D. D. Ganji and A. Sadighi, "Application of He's homotopyperturbation method to nonlinear coupled systems of reactiondiffusion equations," International Journal of Nonlinear Sciences and Numerical Simulation, vol. 7, no. 4, pp. 411-418, 2006.

[6] H. Jafari and S. Seifi, "Homotopy analysis method for solving linear and nonlinear fractional diffusion-wave equation," Communications in Nonlinear Science and Numerical Simulation, vol. 14, no. 5, pp. 2006-2012, 2009.

[7] H. Jafari, M. Zabihi, and M. Saidy, "Application of homotopyperturbation method for solving gas dynamics equation," Applied Mathematical Sciences, vol. 2, no. 48, pp. 2393-2396, 2008.

[8] M. Javidi and A. Golbabai, "A numerical solution for solving system of Fredholm integral equations by using homotopy perturbation method," Applied Mathematics and Computation, vol. 189, no. 2, pp. 1921-1928, 2007.

[9] J. I. Ramos, "Piecewise homotopy methods for nonlinear ordinary differential equations," Applied Mathematics and Computation, vol. 198, no. 1, pp. 92-116, 2008.

[10] Q. Wang, "Homotopy perturbation method for fractional KdV equation," Applied Mathematics and Computation, vol. 190, no. 2, pp. 1795-1802, 2007.

[11] Z. M. Odibat, "A new modification of the homotopy perturbation method for linear and nonlinear operators," Applied Mathematics and Computation, vol. 189, no. 1, pp. 746-753, 2007.

[12] M. Ghasemi, M. T. Kajani, and A. Davari, "Numerical solution of two-dimensional nonlinear differential equation by homotopy perturbation method," Applied Mathematics and Computation, vol. 189, no. 1, pp. 341-345, 2007.

[13] P. Roul and P. Meyer, "Numerical solutions of systems of nonlinear integro-differential equations by homotopy-perturbation method," Applied Mathematical Modelling, vol. 35, no. 9, pp. 4234-4242, 2011.

[14] J. Singh, P. K. Gupta, K. N. Rai, and CIMS-DST, "Homotopy perturbation method to space-time fractional solidification in a finite slab," Applied Mathematical Modelling, vol. 35, no. 4, pp. 1937-1945, 2011.

[15] K. A. Gepreel, "The homotopy perturbation method applied to the nonlinear fractional Kolmogorov-Petrovskii-PISkunov equations," Applied Mathematics Letters, vol. 24, no. 8, pp. 14281434, 2011.

[16] J.-H. He, "Homotopy perturbation technique," Computer Methods in Applied Mathematics and Engineering, vol. 178, no. 3-4, pp. 257-262, 1999.

[17] J.-H. He, "Approximate analytical solution for seepage flow with fractional derivatives in porous media," Computer Methods in Applied Mechanics and Engineering, vol. 167, no. 1-2, pp. 57-68, 1998.

[18] B. Ghazanfari, A. G. Ghazanfari, and M. Fuladvand, "Modification of the homotopy perturbation method for numerical solution of nonlinear wave and system of nonlinear wave equations," The Journal of Mathematics and Computer Science, vol. 3, no. 2, pp. 212-224, 2011.
[19] S. Momani and Z. Odibat, "Homotopy perturbation method for nonlinear partial differential equations of fractional order," Physics Letters A, vol. 365, no. 5-6, pp. 345-350, 2007.

[20] Z. Odibat and S. Momani, "Numerical methods for nonlinear partial differential equations of fractional order," Applied Mathematical Modelling, vol. 32, no. 1, pp. 28-39, 2008.

[21] E. H. Doha, A. H. Bhrawy, and S. S. Ezz-Eldien, "A new Jacobi operational matrix: an application for solving fractional differential equations," Applied Mathematical Modelling, vol. 36, no. 10, pp. 4931-4943, 2012.

[22] Z. Odibat and S. Momani, "The variational iteration method: an efficient scheme for handling fractional partial differential equations in fluid mechanics," Computers \& Mathematics with Applications, vol. 58, no. 11-12, pp. 2199-2208, 2009.

[23] A. A. Hemeda, "Variational iteration method for solving wave equation," Computers \& Mathematics with Applications, vol. 56, no. 8, pp. 1948-1953, 2008.

[24] A. A. Hemeda, "Variational iteration method for solving nonlinear partial differential equations," Chaos, Solitons and Fractals, vol. 39, no. 3, pp. 1297-1303, 2009.

[25] A. A. Hemeda, "Formulation and solution of $n$ th-order derivative fuzzy integrodifferential equation using new iterative method with a reliable algorithm," Journal of Applied Mathematics, vol. 2012, Article ID 325473, 17 pages, 2012.

[26] A. A. Hemeda, "New iterative method: application to the nthorder integro-differential equations," Information B, vol. 16, no. 6, pp. 3841-3852, 2013.

[27] A. A. Hemeda, "Variational iteration method for solving nonlinear coupled equations in 2-dimensional space in fluid mechanics," International Journal of Contemporary Mathematical Sciences, vol. 7, no. 37, pp. 1839-1852, 2012.

[28] K. B. Oldham and J. Spanier, The Fractional Calculus, Academic Press, New York, NY, USA, 1974.

[29] I. Podlubny, Fractional Differential Equations, Academic Press, New York, NY, USA, 1999.

[30] A. A. Hemeda, "Homotopy perturbation method for solving systems of nonlinear coupled equations," Applied Mathematical Sciences, vol. 6, no. 96, pp. 4787-4800, 2012.

[31] A. A. Hemeda, "Homotopy perturbation method for solving partial differential equations of fractional order," International Journal of Mathematical Analysis, vol. 6, no. 49, pp. 2431-2448, 2012.

[32] A. A. Hemeda, "New iterative method: an application for solving fractional physical differential equations," Abstract and Applied Analysis, vol. 2013, Article ID 617010, 9 pages, 2013. 


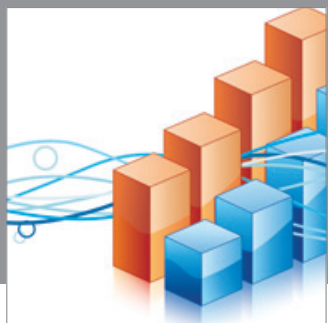

Advances in

Operations Research

mansans

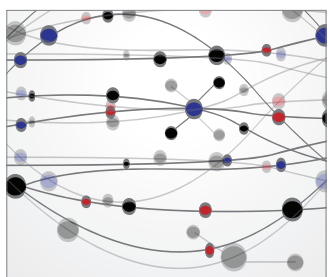

The Scientific World Journal
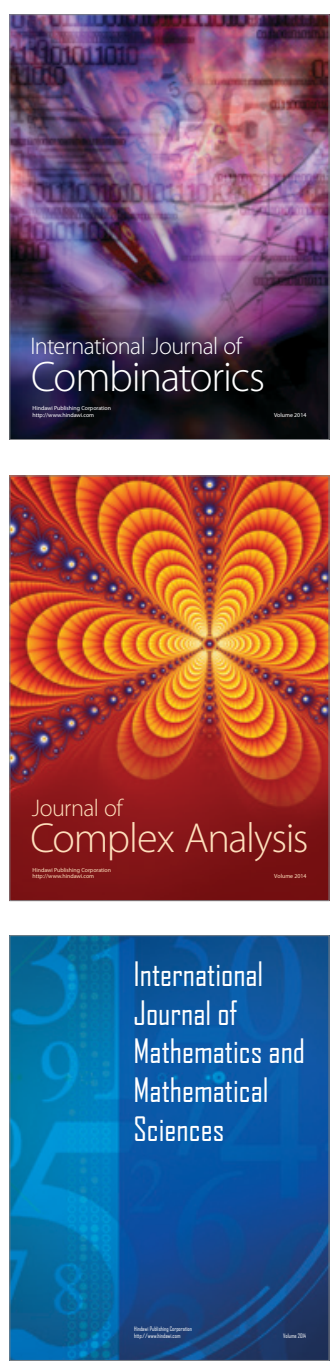
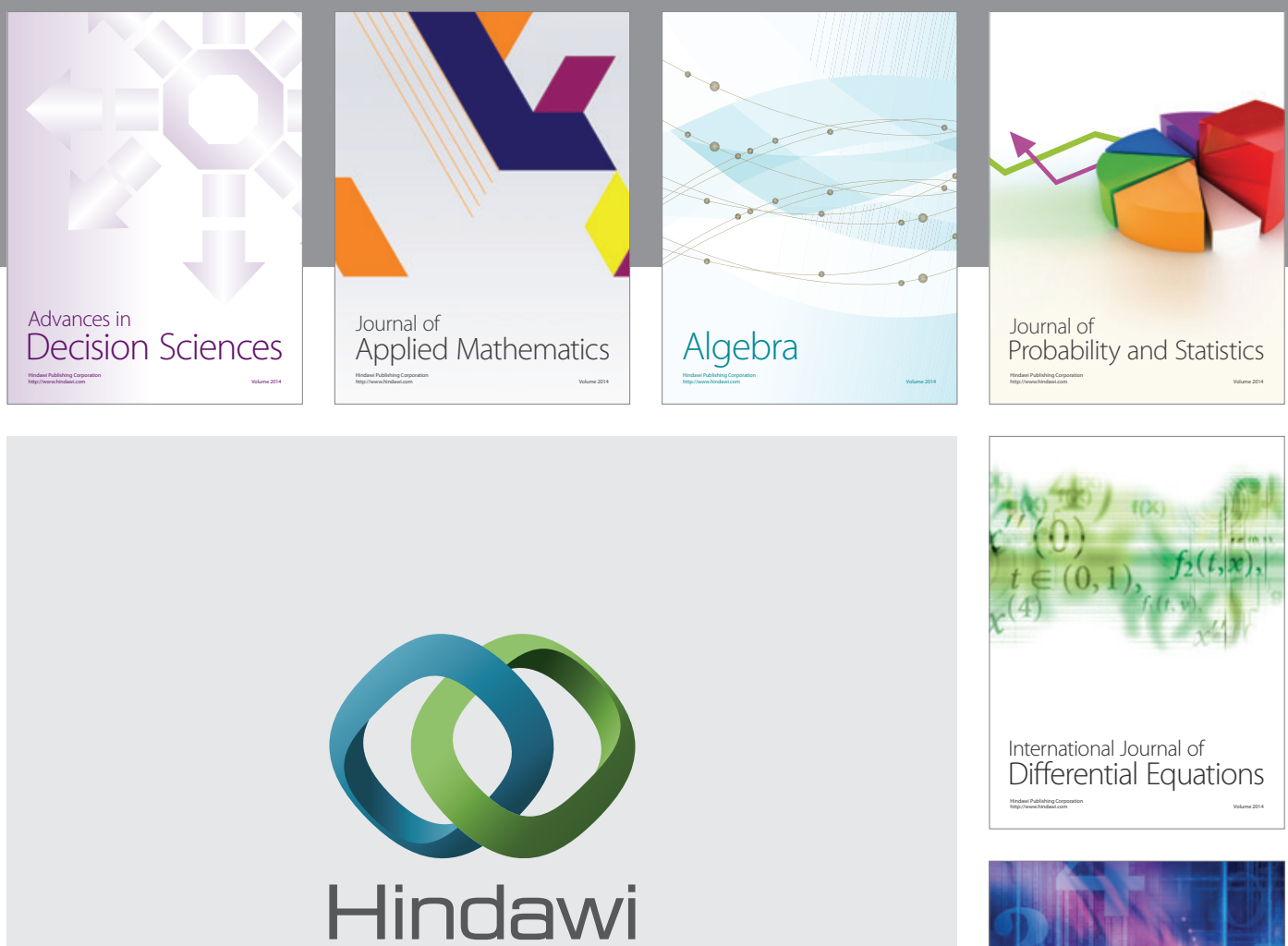

Submit your manuscripts at http://www.hindawi.com
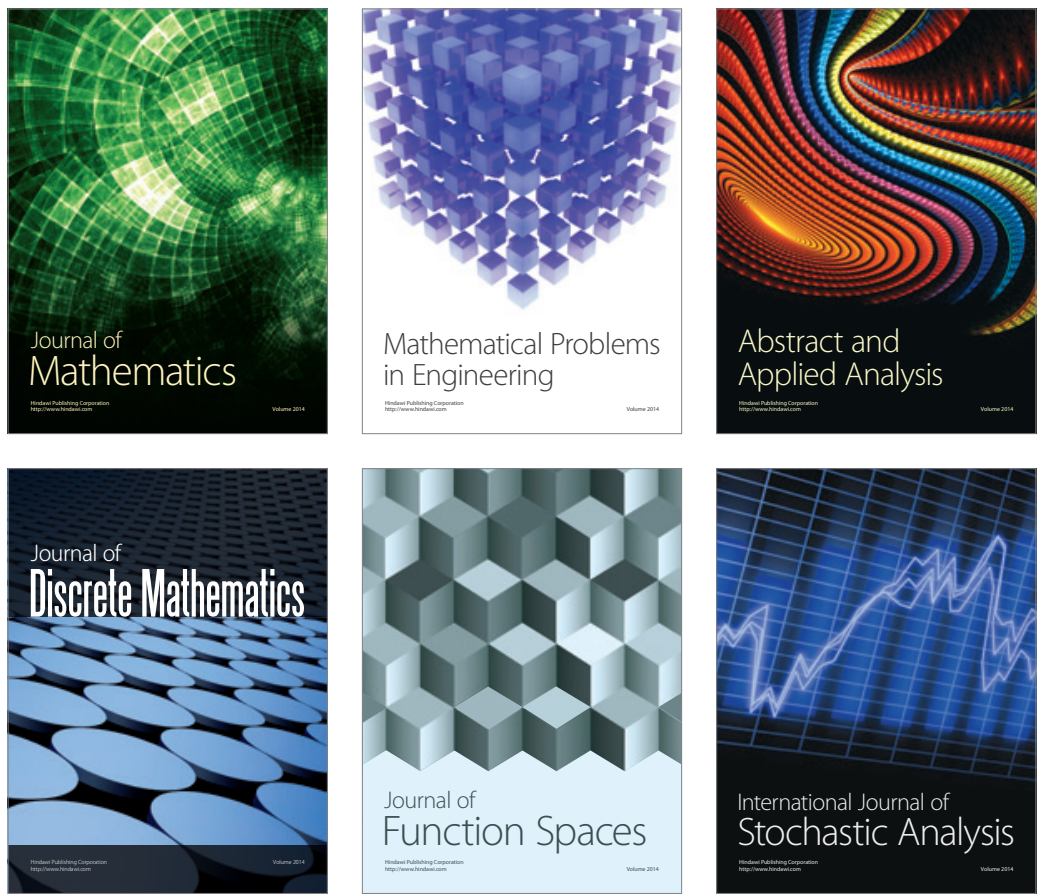

Journal of

Function Spaces

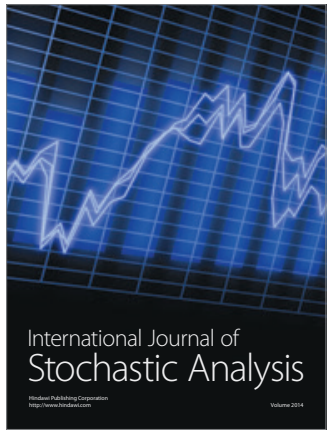

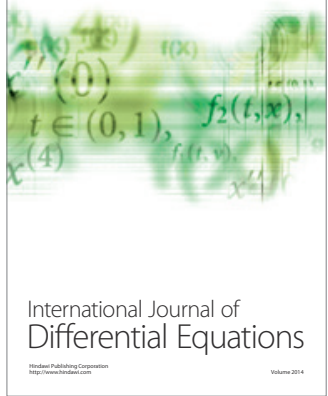
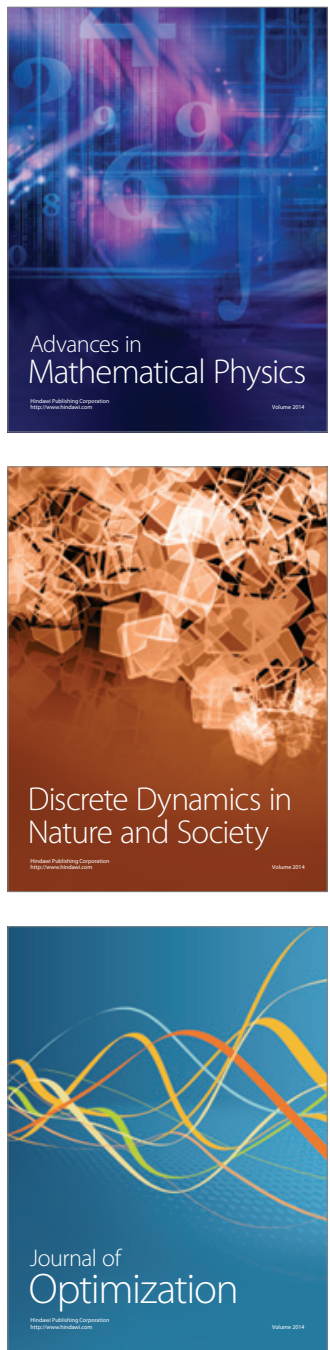